\title{
A propósito de La Dialéctica Concreta de Herbert Marcuse
}

\section{Blanca Muñoz}

Universidad Carlos III de Madrid, Madri, Espanha

\section{Presentación}

La Ontología, según Marcuse, concebida como un conjunto de hipóstasis dogmáticas, desprovistas de cualquier referencia a los conceptos funcionales que rigen las relaciones sociales, es la culpable de la decadencia de un cierto tipo de pensar filosófico. Mas el grave problema es la identificación entre tal pensar y la constitución de la Filosofía. Identificación que está en la base, más o menos "científica", hecha desde el Neopositivismo lógico y la Filosofía Analítica a todos aquellos que, desde distintas actitudes teóricas, han intentado llevar a cabo una investigación pormenorizada sobre las mutuas interrelaciones entre mente y realidad, entre concepto y acción.

Marcuse precisa, en este sentido, la necesidad actual de la Ontología, pero de una forma de Ontología que sea al mismo tiempo una ruptura con la concepción tradicional abstracta y especulativa y en las formas neopositivistas. Para el autor de "Über konkrete Philosophie" hay que volver a la realidad natural, hacia lo que aparece como "dado", como fundamentación subyacente de la vida del sujeto. La Ontología no puede seguir engañándose al respecto de aquello que puede ser considerado como lo real objetivo. Marcuse criticará a Heidegger precisamente por su inconsecuencia ante el primado del "no-yo" frente al "yo", ante la prevalencia de lo abstracto sobre lo concreto. Piensa Marcuse que el sujeto debe restaurarse en el centro del movimiento dialéctico, pero no el sujeto abstracto, el sujeto heideggeriano El sujeto que conforma el Universo según el orden de su pensar y no delimita las posibilidades de tal pensar, ni siquiera incluso si este movimiento universal posee una serie de leyes distintas al movimiento lógico de la racionalidad humana. Esta pretensión egocéntrica está en el origen de la Filosofía que no toma parte por una dialéctica negativa ${ }^{1}$. Por el motor de la negatividad como eje a partir del cual comprender el sentido de la realidad, y mientras se siga manteniendo la ilusión del sujeto abstracto, la irracionalidad condicionará, como una espada pendiendo sobre las cabezas de la especie, el rumbo de la Historia.

La reflexión ontológica de Marcuse se sitúa entonces en el origen de su creación teórica. Unos años antes de incorporarse al naciente "Instituto de Investigación Social", cuyo año de incorporación de Marcuse fue en el fatídico 1933, la creación del autor berlinés estará 
marcada por la influencias de Husserl y Heidegger ${ }^{2}$. El primer Marcuse se acerca a la Ontología con la finalidad de llegar a una teoría de la Historicidad ampliando los planteamientos de Hegel. La consecuencia de este análisis llevará al pensador alemán hacia el Marxismo y, sobre todo, a la reflexión ontológica sobre la realidad histórica. Fruto de estos años serán fundamentalmente los siguientes estudios: "Beiträge zu einer Phänomenologie des historischen Materialismus" ("Contribuciones a una fenomenología del Materialismo Histórico") (1928), "Zur Wahrheitsproblematik der soziologischen Methode" ("El problema de la realidad histórica") ("Para la problemática de la verdad del método sociológico") (1929), "Neue Quellen zur Grundlegung des historischen Materialismos" ("Nuevas fuentes para la fundamentación del Materialismo Histórico") (1932), "Uber die philosophischen Grundlagen des writschaftswissenschaftlichen Arbeitsbegriff" ("Sobre los fundamentos filosóficos del concepto científico-económico del trabajo" (1933), entre estos primeros trabajos estará "Uber konkrete Philosophie" (1929). La gran mayoría de ellos se publicarán en Archiv für Sozialwissenschaft und Sozialpolitik y en Die Gesellschaft, dos publicaciones que gozaban de un alto y reconocido prestigio por la influencia que Max Weber, Werner Sombart y Georg Simmel tuvieron en su creación.

La preocupación marcusiana por relacionar Ontología e Historicidad culminará durante estos años en la búsqueda de una conexión entre Fenomenología y Dialéctica. Marcuse experimentará la atracciónrechazo de la influencia de Heidegger. No podemos olvidar que en 1923 Marcuse realizará su tesis de habilitación docente "Hegels Ontologie und die Grundlegung einer Theorie der Geschichtlichkeit" ("La Ontología de Hegel y la fundamentación de la Historicidad" (1932) bajo la dirección de Heidegger. La desavenencia de Marcuse con Heidegger provendrá de la interpretación que sobre "el ser del sujeto" mantendrán ambos autores. El sujeto abstracto y más irracionalista de Heidegger restaura como ontológico un mundo del "ser" que presenta las deficiencias de una realidad inexistente $y$, únicamente, válida para su conciencia. Esta pretensión termina, como en Kierkegaard, en el salto en el vacío, en un sujeto desprovisto de sus interrelaciones, o también en un nirvana artificial y pretencioso. Marcuse crítica de este modo tanto el existencialismo heideggeriano como el irracionalismo idealista de Kierkegaard. En las dos posiciones el miedo ontológico a la realidad natural, y sobre todo a sus consecuencias, erige en sistema la reflexión subjetiva, y a partir de ella determina el orden de la realidad, la sucesión de "las cosas naturales". Así, la Ontología tendrá que reencontrar su propio sentido en la dialéctica entre sujeto y objeto, entre pensar y actuar. Para Marcuse, la reflexión primera acerca del "ser" tiene que iniciarse 
desde la negatividad de ese mismo "ser". No hay otra posibilidad si se quiere conservar aún la honestidad de la Filosofía.

La Ontología, pues, ha de partir siempre de la negatividad, del análisis de los fenómenos entendidos bajo la perspectiva de su propia negación. La positividad genera un estado de inmutabilidad, de falsa quietud imposible de ser comprendida en su total automovimiento. Marcuse, como Hegel y como Marx, entiende el problema de la realidad a partir del movimiento, de la mediación, del devenir. Captar el sentido profundo de los fenómenos, que no constituyen un fenomenismo en sentido humeano, desde la dialéctica es el sentido que tiene hoy la tarea de la Ontología. Pero esa Ontología ha de ser necesariamente negativa. La negación contiene en sí el paso hacia "otro estado de cosas" y ello, rigurosamente planteado, es el origen de la actitud crítica, entendiendo por actitud crítica aquella que huye del dogmatismo y de la rigidez conceptual. Ningún análisis más centrado en el estudio de la realidad que el llevado a cabo por Marcuse. La realidad posee, por tanto, intrínsecamente un factor de cambio que la determina en su totalidad. $Y$ es este factor de cambio el que tiene que ser subsumido en las categorías conceptuales de la Ontología que quiera presentarse como testigo desapasionado, o apasionado si por tal se entiende el deseo de transformación y de modificación estructural. A ese propósito consagra Marcuse su necesidad de Ontología. Frente a la postura del Neopositivismo, o de la Filosofía Analítica, la misión de la Filosofía es llevar a cabo una síntesis crítica de todos los aspectos de lo real sin omitir ninguno por pequeño o accesorio que sea. La necesidad de Ontología, en este sentido, es una necesidad revolucionaria. Es una necesidad de la razón crítica, y por ello mismo tal Ontología tiene que contener a la negatividad como su imprescindible motor histórico. Marcuse, como Adorno, percibe el avance de la Filosofía, como pensar teórico y práctico, dentro de un contexto de necesidades humanas sentidas y deseadas por los grupos e individuos que anhelan el avance progresivo de la Historia. Realidad e Historia, entonces, coinciden cuando la dialéctica como crítica negativa busca, ante todo, el desarrollo del auto-movimiento de lo real, pero no un desarrollo sometido a las reglas anárquicas del azar. Al contrario, la Dialéctica negativa fundamenta así una Ontología basada en la voluntad y en la misma acción racional de los seres humanos. Es la dirección de la Historia desde la razón. Sería el sueño hegeliano de la realización y culminación de la Historia percibido como el camino colectivo hacia la libertad.

\section{La dialéctica de lo concreto.}

En 1929 Marcuse aún muestra las influencias de Husserl y de Heidegger en su análisis de lo real. La Fenomenología había insistido de manera especial en el carácter objetivista del "ser". Sin embargo, 
el carácter subjetivo del planteamiento de Husserl no puede soslayarse en el tema del reduccionismo ontológico que queda sometido a unas abstracciones fundamentalmente de índole lógica. A este respecto, el subjetivismo, cuanto postura ontológica, tiene que refugiarse en la cada vez más evanescente concepción abstracta del ser de lo real. Husserl, de manera paradójica, comenzó su Fenomenología como una indagación objetivista acerca del "ser" de la realidad, pero en el mismo fondo de la Fenomenología latía inexorablemente el subjetivismo del "Cogito" cartesiano. Así, Husserl tuvo que ir realizando sublimaciones de la facticidad de los fenómenos. Las reducciones husserlianas tendieron a privar de significado determinado no sólo a las existencias individualizadas sino, a la par, a las abstracciones racionales. El concepto de "ser" pasó de este modo a considerarse una tautología. Y si el "ser" desaparece por innecesaria sublimación, también el sujeto se disuelve como una abstracción carente de sentido fenomenológico y óntico.

En el mismo sentido, el sujeto pasa a convertirse en un asunto evanescente en la ontología heideggeriana. Como afirma Kart Heinz Haag: “... ya Hegel demostró que hasta el juicio analítico, lo quiera o no, requiere por su misma naturaleza la predicación de lo que no es simplemente idéntico con el mero concepto de sujeto." La búsqueda de un fundamento objetivo que garantice no únicamente al sujeto cuanto la existencia de la realidad, conduce al objetivismo ontológico que surge en el mismo momento en el que se percibe esta realidad como una suma de aspectos contrapuestos, como una dialéctica en la que sus propias determinaciones son las causas efectivas de la explicación ontológica.

La dialéctica, pues, aparece como camino seguro hacia la fundamentación gnoseológica y ontológica del "ser del sujeto" y "del ser del objeto". A este respecto, el siglo XIX aportó el camino transitable seguro de la Ontología. Con Hegel, el platonismo de las ideas enfrentadas a un mundo de objetos, que necesariamente tienen que participar de las propias ideas para poder garantizar su propia existencia, queda abolido. Pero frente al idealismo platonizante retomado de nuevo por Husserl y Heidegger no puede afirmarse otra solución que la explicación dialéctica. La Teoría Crítica, como Ontología objetivista, hace que la "Idea" clame por realizarse, tal y como afirmaría Hegel. Pero una crítica realizada prácticamente exigirá una transformación integral de la realidad en su conjunto. Sin embargo, la revolución sólo como cambio político no sirve más que para la sustitución de los dominadores por otros de idéntico signo y, ahora, hacia lo que habrá que aspirar será hacia una liberación universal del ser humano, y a esta tarea consagrará de manera precisa Marcuse su creación intelectual y vital.

Marcuse recobra así la dialéctica de Marx frente a la disolución de la existencia en una Ontología abstracta del "yo". Por ello, se hace 
esencial la publicación de "Über konkrete Philosophie" escrita en el año 1929. En este estudio se encuentra el giro posterior que Marcuse va a emprender cuando empieza a tomar contacto con la naciente Teoría Crítica. El tema esencial de este estudio girará sobre qué debe ser una "philosophische konkrete" que conlleva, ante todo, dos temas sin los cuales no sería posible la fundamentación de la Teoría Crítica: la idea de totalidad en lo ontológico y social y la concepción de lo verdadero como praxis.

Marcase enlaza la Fenomenología y el Marxismo a partir de una nueva concepción de "Lebenwelt". El "mundo-de-la-vida" tiene que ser considerado como totalidad de las esferas sociales que actúan en él. Pero la gran revolución marcusiana de "Lebenwelt" dimana del acercamiento que Marcuse imprime al interrelacionar el abstracto "mundo-de-la-vida" con la cotidianidad; esto es, con lo concreto, con lo que se desarrolla en lo efectivo. En lo que conduce hacia la totalidad concreta, a la que se refería Lukács, sólo que ahora el problema de "lo concreto" no puede desvincularse ni de la esfera pública (la publicidad en el significado de Habermas), pero tampoco de la facticidad y de lo realizable. La consecuencia de ello será la inserción de tal "mundo-de-la-vida" en la concepción de la Teoría Crítica como aclaración racional de la realidad, y desde esta posición el "Lebenwelt" deja de ser "realidad-en-sí" para mostrarse en su totalidad como "realidad-para-nosotros".

En efecto "Ubre konkrete Philosophie" tiene el determinante significado de constituir la investigación que aclara no simplemente a su autor cuando, también, a sus lectores en la dirección de establecer el rompecabezas con el cual se da un cariz nuevo a la comprensión ontológica de lo real. Lo real será fundamentalmente histórico, y no hay que olvidar en este punto que Marcuse ha realizado su Tesis sobre una Ontología de la Historia, siguiendo en ello a un Hegel que centra en el Espíritu, o en la conciencia del tiempo, la Fenomenología de una dialéctica de la conciencia infeliz. Y es sobre ella sobre la que la Fenomenología de Husserl se ve superada en Marcuse por la Fenomenología hegeliana, y en la que la Historia se desenvuelve en la conciencia determinada por el movimiento de los acontecimientos sociales. La filosofía concreta no puede dejar de ser sino la suma del "ser" y de la "Historia". Heidegger, Marx, Husserl y Lukács son armonizados en una totalidad que abarca "la cotidianidad de la conciencia infeliz". No es de extrañar que su adscripción al Instituto de investigación Social se produzca al año siguiente de la publicación de su reflexión sobre la filosofía concreta y, tampoco, es insólito que sus siguientes escritos se empiecen a centrar en el autoritarismo ("Der Kampf gegen den Liberalismos in der totalitären Sttaatsauffassung") y en el tema permanente, en el análisis marcusiano, de la liberación y sus causas ("Zur Kritik des Hedonismus"). 
Las imposiciones políticas de la época no pueden olvidarse en la conformación intelectual de Marcuse. Si en "Über konkrete Philosophie" se aboga por una Ontología de lo manifiesto y se preludian sus posteriores intereses por la cosificación y el fetichismo que van a desembocar en las grandes contribuciones del "Marcuse maduro": "El hombre unidimensional" y "La agresividad en la sociedad industrial avanzada", no hay que desatender el caminar teórico de Marcuse hacia el análisis psico-ontológico de Freud y el Psicoanálisis. Y por tanto, si la filosofía concreta se dirige con pasos de gigante hacia lo público, en 1931 en "Das Problem der geschichtliche Wirklichkeit" se manifiesta ya el nuevo rumbo que la relación con los frankfurtianos, Horkheimer y Adorno, va a imprimir a sus intereses teóricos: la transformación revolucionaria del pensamiento y de la sociedad.

En conclusión, la publicación al portugués dirigida por el Doctor Jorge Coelho Soares de "Über konkrete Philosophie" profundiza el conocimiento complejo de la obra del autor de "Eros y civilización". El "primer Marcuse" nos aporta aquí el conocimiento de los hilos con los que tejió el monumental tapiz de su obra. Vemos en sus primeros estudios "Beiträge zu einer Phänomenologie des historischen Materialismus Methode" (1929) y, esencialmente, en "Ubre konkrete Philosophie" (1929) como Marcuse renueva la Ontología heredada fenomenológicamente a partir de la restauración de un Materialismo Histórico heterodoxo de influencias lukácsianas. Lo concreto, así, asumirá su lugar privilegiado no sólo en el Marxismo, sino en el Marxismo-freudiano con el que Marcuse se acerca a los individuos desde sus complejidades psicológicas y sociales. La filosofía de lo concreto resulta el punto de inflexión para comprender el trayecto y las direcciones que la Teoría Crítica va a asumir por influencia esencial de Marcuse. En esta obra ya encontramos en germen las intuiciones ontológicas que florecerán años después en la etapa norteamericana de Marcuse tanto de su paso por la Universidad de Columbia como en sus últimos años en California. Lo concreto no puede desvincularse de la transformación del mundo. El final de la Utopía lleva a cambiar la Historia para cambiar la Vida, como decía la frase de Rimbaud, y es, sobre todo, en lo concreto, y en la transformación revolucionaria de lo concreto, en donde los ideales de cultura, justicia y libertad son retomados por Marcuse en el sentido de Rimbaud: para lograr la posibilidad real de cambiar la unidimensionalidad de la vida, haciendo concretas las posibilidades utópicas y objetivas de la Historia.

\section{Referencias}

HAAG, K. H. Kritik der neueren Ontologie. Stuttgart, 1960. p. 73. MARCUSE, $\mathrm{H}$. Ontología de Hegel y teoría de la Historicidad. La Tesis de Marcuse para la venia docendi, Barcelona: Editorial Martínez 
Roca, 1970. (“Hegels Ontologie und die Grundlegung einer Theorie der Geschichtlichkeit"). . Las Obras Completas. En: Schriften. Frankfurt/Main: Suhrkamp, 1978-1987. Nueve Volúmenes.

\section{Endereço para correspondência}

Blanca Muñoz

Universidad Carlos III de Madrid, C/. Madrid, 126, 28903 Getafe, Madrid, Espanha

Endereço eletrônico: bmunoz@polsoc.uc3m.es

\section{Notas}

${ }^{1}$ La mejor investigación sobre este tema en: Adorno, Th. W.: "Dialéctica Negativa". Madrid: Taurus, 1975. Obras Completas: “Gesammelte Schriften". Suhrkamp Verlag, Frankfurt am Main, 1973. Veintitrés Volúmenes a cargo de Gretel Adorno y Rolf Tiedemann.

${ }^{2}$ MARCUSE, H. Über konkrete Philosophie. Frankfurt am Main, Europäische Verlagsanstalt, 1973. Y en: "Schriften". Vers. cit. 1987. Un análisis bastante interesante en: Spiro, L.: "The Freudo-Marxism of Herbert Marcuse". Columbia University Press, 1973. 